\title{
"Zur Denaturalisierung“. Wissenschaftlerinnen und Akademikerinnen in Spanien
}

Studien über den Zugang von Frauen zur wissenschaftlichen Laufbahn in Spanien haben eine lange Geschichte. Sie zeigen, dass Frauen seit dem ersten Drittel des zwanzigsten Jahrhunderts sowohl in wissenschaftlichen Studiengängen als auch in den Wissenschaftskarrieren, in Hochschulforschung und -lehre dauerhaft und zunehmend präsent sind. Seit Frauen 1910 zum Hochschulstudium zugelassen wurden, beteiligen sie sich sowohl an den experimentellen Wissenschaften als auch an den Geisteswissenschaften (Muñoz Repiso 1988; Magallón 1998; Santesmases 2001). Das Vorurteil, dass Frauen eher den „Geisteswissenschaften“ als den „Naturwissenschaften“ zuneigen, blieb dennoch bestehen und spiegelt sich bis heute in der entsprechenden Verteilung von Mädchen und Jungen in der Sekundarschule und auf dem Gymnasium sowie im niedrigen Anteil von Mädchen in Ingenieursschulen wider. Geschlechterstereotype scheinen sowohl in der gesamten Geschichte der Wissenschaft und der Universitäten als auch in den historischen Rekonstruktionen dieser Geschichte allgegenwärtig zu sein (Alcalá Cortijo 2002, 2006). In der Wissenschafts- und Technikgeschichte blieben Frauen weitgehend unsichtbar. Dabei müssten doch besonders diejenigen, die die Arbeit in den Laboren, Krankenhäusern und Universitätshörsälen kennen, auf dieses permanente Unsichtbarmachen der dort tätigen Frauen, aufmerksam werden.

Soziales Verhalten wurzelt in Geschichte. Gesellschaften, die nach Geschlechtern segregiert sind und Praktiken der Diskriminierung entstehen nicht aus dem Nichts. Frauen und ihre kulturellen Räume können deshalb nicht singulär, sondern nur in der Beziehung zu anderen analysiert und definiert werden. Die kulturelle Rolle der Frau wird immer in Bezug auf die Rolle des Mannes und auf bestimmte Begriffe wie Macht, Autorität und Beziehung gedacht. Die Geschichte der Frauen wird auch aus der Sicht von Frauen selbst rekonstruiert. Untersuchungen über Wissenschaftlerinnen und Akademikerinnen in Spanien wurden hauptsächlich von Frauen durchgeführt. Dies waren Forscherinnen, die nicht nur die weibliche Präsenz in den Berufen außerhalb des Hauses sichtbar machten, sondern auch historiographische und intellektuelle Genealogien und Bezugspunkte für zukünftige Studien erarbeitet haben.

Solche Studien über Frauen, die Wissenschaften und das Geschlecht erschienen in Verbindung mit der wiederauflebenden feministischen Bewegung in Spanien und müssen zu dieser in Beziehung gestellt werden (Scanlon 1986). Seit den 1980er Jahren ist die akademische Gruppe, die sich der Geschlechterforschung widmet, stark gewachsen. Institute für feministische Studien an spanischen Uni- 
versitäten, statistische Untersuchungsreihen und neuerdings gesetzliche Regelungen zur Verhinderung von Diskriminierung stellen Wissen zur Verfügung und beeinflussen die feministische Kultur, die Lebensbedingungen von Frauen in Spanien sowie die Agenda der Genderpolitik der gegenwärtigen Regierung. Im Ergebnis wächst das Bewusstsein über die Geschlechterverhältnisse sowohl in politischen Entscheidungsprozessen als auch in Untersuchungen und Forschungen über Frauen. Daraus resultiert eine Vielzahl von neuen Beiträgen, angefangen bei den Arbeiten, die junge Forscherinnen in feministischen, soziologischen, geschichtswissenschaftlichen und medizinischen Studiengängen in Spanien veröffentlichen, bis hin zu Biographien über Wissenschaftlerinnen und Studien über das Geschlecht des Wissens in Experimental- und Gesundheitswissenschaften sowie in sozialen und politischen Praktiken. Hier entfaltet sich eine Kultur, die zur Entlarvung des Androzentrismus beiträgt, sowohl in der empirischen Forschung als auch in den Sozial- und Kulturwissenschaften.

Auf dem Weg zu einer Welt mit sozial gleichen Subjekten sind jedoch weiterhin zahlreiche Barrieren aufgerichtet. Obwohl die Welt schon sehr vielversprechend aussieht, ist sie doch durch Segregation und Diskriminierung charakterisiert, sowohl vertikal als auch horizontal. Dies zeigen nicht nur Statistiken über (wenige) Frauen in Führungspositionen, sondern auch die Tatsache, dass akademische Anerkennung für Frauen noch auf die Geschlechterforschung in den Universitäten beschränkt ist.

Im Folgenden möchte ich einige Gedanken über diese z.T. widersprüchlichen Entwicklungen vorstellen. Frauen, die sich in Spanien der Frauen- und Geschlechterforschung widmen, studieren in gewisser Weise sich selbst. Sie erforschen ihre eigene Genealogie auf der Suche nach Anerkennung für diejenigen, die bis vor kurzem in der Geschichtsschreibung und in der zeitgenössischen Fachliteratur verborgen geblieben sind. Unter diesen Umständen ist die Präsenz von Frauen sowohl in der akademischen Welt als auch in der feministischen Forschung doppelt versteckt: Sowohl die Autorinnen als auch ihre Studienobjekte bleiben im Verborgenen. Beispielsweise sind die medizinischen Praktiken von Frauen, wie die von Heilerinnen und Hebammen, nicht sichtbar geworden, ebenso wenig wie die Frauen, die Arbeiten über diese Frauen verfasst haben (Cabré / Ortiz Gómez 2001). Diese Leerstelle erscheint heute jedoch von akademischen Intellektuellen und ihren Werken kompensiert zu werden, sowohl auf der politischen Agenda als auch in der sozial-beruflichen Praxis. Der folgende Beitrag bietet daher ein Panorama der neueren Arbeiten zur akademischen Frauen- und Geschlechterforschung. Dabei ist es wichtig, den Zusammenhang zwischen Frauenforschung einerseits, d.h. Recherchen zur Beteiligung von Frauen und Biographien zu Wissenschaftlerinnen und Forscherinnen, und Geschlechterforschung andererseits im Auge zu behalten. 


\section{Zur Geschichte von Wissenschaftlerinnen in Spanien}

Schriftstellerinnen, Pädagoginnen, Philosophinnen, Juristinnen, Physikerinnen, Chemikerinnen, Mathematikerinnen, Heilerinnen, Hebammen und Ärztinnen sind seit Jahrzehnten tätig. In Bezug auf die Geschichte der weiblichen akademischen Professionalisierung in Spanien wurde ihnen nicht nur ausdrücklich der Zugang zu Wissen verweigert, sondern auch der Beitrag von Frauen zur Entwicklung der Profession unterschlagen. Zum hundertjährigen Jubiläum des Gesetzes, das Frauen den Zugang zum Hochschulstudium in Spanien erlaubte, wurde auch daran erinnert, dass Frauen häufig abgeraten wurde, ein Hochschulstudium zu beginnen. Nur wenige Frauen hatten Zugang zu den Universitäten, bevor ihre Aufnahme 1910 ausdrücklich per Gesetz erlaubt wurde. Erst diese Rechtsnorm ermöglichte die Anwesenheit von Frauen in den Hörsälen. Und obwohl die spanische Gesellschaft der damaligen Zeit durch vorwiegend ländliche Struktur und sozialen Schichtung gekennzeichnet war, erhöhte sich die Zahl der Studentinnen bis zur zweiten Republik (Capel 1982; Flecha 1996; Zulueta 1984; Zulueta / Moreno 1993). Die Zulassung zu höherer Bildung führte auch dazu, den (gesellschaftlichen) Status von Frauen besser zu verstehen. Bildung kann dazu beitragen, Verhalten zu formen und die Rollenverteilung im Haus wie in der Öffentlichkeit neu auszurichten. Bildung ermöglicht außerdem den Zugang zu qualifizierten Arbeitsplätzen und Berufslaufbahnen, so dass Frauen mittlerweile auch in höheren Ebenen der Arbeitsgesellschaft präsent sind (Salido 1996). Darüber hinaus hat die Alphabetisierung von Frauen nachweislich einen Einfluss auf die wirtschaftliche Entwicklung in Spanien gehabt (Núñez 1992). Zwischen der Alphabetisierung und dem allgemeinen Zugang zur Hochschulbildung liegt eine Zeitperiode, in der die kulturellen Barrieren des Zugangs zur Hochschulbildung für Frauen größer waren als für Männer. Zunächst aber war die soziale Klasse für den Hochschulzugang wichtiger als das Geschlecht, so dass Frauen der Oberschicht eher studieren konnten als Männer aus einer Klasse mit geringeren ökonomischen Mitteln und anderem kulturellem Niveau (Durán 1972; Carabaña 1984 und 1997).

Von 1915 bis 1916 studierten 13,1 Prozent der Studentinnen und von 1932 bis 1933 20,4 Prozent Naturwissenschaften. Der Anstieg ist signifikant und deutet auf einen Trend hin, der durch den Bürgerkrieg (1936-1939) und durch die diskriminierenden Vorschriften während der Diktatur von General Francisco Franco (1939-1975) durchbrochen wurde. Dies betraf sowohl die naturwissenschaftlichen Studien (Physik, Chemie und Mathematik) als auch die ersten Lehrgänge der Medizin und Pharmazie. In diesen Studiengängen hatten sich junge spanische Frauen in höherem Maße eingeschrieben als in anderen Hochschulfächern (Magallón 1998; Santesmases 2001). Seit 1964 ist zu beobachten, dass der Anteil von Frauen mit einem Hochschulabschluss in Spanien rasch wächst. Diese Zunahme zeigt zugleich auch eine geschlechtliche Umverteilung innerhalb der Studiengänge, die bisher überwiegend von Männern studiert wurden, wie Me- 
dizin, Jura oder Naturwissenschaften. Mitte der 1960er Jahre überstieg die Zahl der Studentinnen und Absolventinnen die 30-Prozent-Hürde, bevor die Gesamtzahl aller Studenten insgesamt sehr stark zunahm. Dies war allerdings keine Folge neuer Studienplätze an den 1968 gegründeten Universitäten in Bilbao, Madrid und Barcelona. Vielmehr waren diese Neugründungen eine Reaktion auf das wachsende Interesse an höherer Bildung, das die franquistische Politik als „Vermassung“ diskreditierte und - im Jahre der europäischen Studentenunruhen - entsprechend repressiv einzuhegen versuchte. Dieses Interesse wiederum ist letztlich auf das Wirtschaftswachstum in Spanien zurückzuführen, das im Jahrzehnt zuvor begonnen hatte und dazu beitrug, auch die Bildungsansprüche der Eliten zu ändern (Santesmases 2001). Der Prozentsatz der weiblichen Absolventen in allen wissenschaftlichen Fächern - inklusive Naturwissenschaft, Medizin und Pharmazie - lag im Studienjahr 1969/70 bei 43,3 Prozent, der Prozentsatz der weiblichen Absolventen in Philosophie und Literatur lag bei 44,6 Prozent.

Seit 1969 waren Frauen zu fast gleichen Teilen an den Natur- und Geisteswissenschaften beteiligt. Dies korrespondierte mit der wachsenden Zahl von Frauen, die in den Rechtswissenschaften ihren Abschluss machten, einem Studiengang, der historisch „,männlich“ konnotiert war. So ergab sich eine größere Beteiligung spanischer Studentinnen in allen akademischen Bereichen. Dies führte auch zu einer Veränderung der typisch „männlichen“ oder „weiblichen“ Studiengänge, wobei sich allerdings die Veränderungen auf weiblicher Seite schneller entwickelten, da die Zahl der weiblichen Absolventen in kürzerer Zeit anwuchs als die der männlichen (Santesmases 2001).

Im Jahrgang 1982 / 83 waren 46,2 Prozent aller Studenten an den spanischen Universitäten Frauen. Sie stellten 47 Prozent aller Studierenden der experimentellen Naturwissenschaften, 49,9 Prozent bei den Gesundheitswissenschaften, 44,5 Prozent bei den Rechts-und Sozialwissenschaften und 63,5 Prozent bei den Geisteswissenschaften. Im Ingenieurswesen blieb der weibliche Anteil bei 11,1 Prozent. Umgekehrt heißt dies, dass 11,7 Prozent aller Studentinnen naturwissenschaftliche Fächer wählten, 19,4 Prozent Gesundheitswissenschaften, 2,2 Prozent Ingenieurwissenschaften, 41,7 Prozent Rechts- und Sozialwissenschaften und 25,1 Prozent Geisteswissenschaften. Dies zeigt, dass Frauen in allen Studienbereichen präsent waren und auch Studienbereiche belegten, die für Frauen ungewöhnlich waren, wie etwa Rechts- und Experimentalwissenschaften. Somit waren Stereotypen aufgebrochen worden (Pérez Sedeño 2005). Im Studienjahr 1990/91 haben Frauen dann bereits 51 Prozent aller Studierenden ausgemacht. In allen Studiengängen waren Frauen in der Mehrheit, außer in den Ingenieurwissenschaften, wo sie nur mit 20 Prozent vertreten waren, und in der experimentellen Wissenschaft (48,1 Prozent).

Während dieser Zeit waren die Zahlen der weiblichen Studienabbrecher sehr niedrig: 1982/ 83 waren 53,7 Prozent und 1990/91 57,8 Prozent der Studienabgänger Frauen. Die Zunahme gegenüber dem Studienjahr 1972 / 73, als dieser 
Prozentsatz bei 31,7 lag, ist sehr wichtig und zeigt die steigende Präsenz von Frauen an den Hochschulen, was sowohl ihre Befähigung als auch ihre Ambitionen belegt (Pérez Sedeño 2005). Im Jahr $2000 / 01$ blieben die Frauen immer noch in der Mehrheit unter den Absolventinnen und Absolventen (57,41 Prozent), obwohl der Prozentsatz seit Studienjahr 1997 / 98 leicht gesunken ist, als er noch bei 59,9 Prozent lag. Zwischen 1997 und 2001 erreichte der prozentuale Anteil der Absolventinnen in den Ingenieurswissenschaften allerdings nicht die 30-Prozent-Marke (Pérez Sedeño 2005).

Im Zeitraum von 1970 bis 1990 ist auch die Zahl der Promotionen von Frauen gestiegen, allerdings ist die Zahl der Doktorandinnen in Relation zu den Zahlen der Hochschulabsolventinnen zurück gegangen: Im Semester 1972/73 haben 21,8 Prozent der Absolventinnen promoviert, 1982/83 waren es 41,2 Prozent und $1990 / 91$ 40,6 Prozent, bis im Jahr 2001 die 50-Prozent-Marke um ein Zehntel überstiegen worden ist. Diese Daten deuten darauf hin, dass hier ein geschlechtsspezifischer Bias besteht, dessen Haupteffekt ist, Frauen von der universitären Weiterqualifikation abzubringen. Diese Schere besteht bis heute in Spanien: Das Bild zeigt auf der einen Seite eine zunehmend höhere Anzahl von Studentinnen als Studenten und auf der anderen Seite eine schrittweise Verringerung der Präsenz von Frauen unter den Promovierenden, die sich für Stipendien zur Ausbildung in Forschung und Lehre qualifizieren und sich damit für stabile berufliche Positionen zwischen dem Forschungspersonal und den Dozenten entscheiden. Und schließlich sind es nur wenige Frauen, die anerkannte akademische Positionen erreichen, nämlich Universitätslehrstühle und Forschungsprofessuren des Nationalen Forschungsrates, der 1971 gegründet wurde.

So haben dennoch der Lauf der Zeit und die Förderung einer nicht-geschlechtsdiskriminierenden Bildung nicht ausgereicht, um den Sexismus in den Naturwissenschaften, der Forschung oder der Universitätslehre zu brechen. Dies führte Paloma Alcalá zu der Aussage, dass die Metapher der ,gläsernen Decke“ durch die vom „,klebrigen Asphalt“ ersetzt werden sollte. Denn bis heute gilt, dass die Präsenz von Frauen in den Bereichen der Macht bei maximal 30 Prozent liegt (Alcalá 2008).

\section{Statistiken und akademischer feministischer Aktivismus}

Diese Ergebnisse führten Maria Antonia García de León, María Luisa García de Cortázar und Paloma Alcalá sowie Eulalia Pérez Sedeño dazu, von einer geschlechtshierarchischen Diskriminierung in Spanien zu sprechen. Als Konsequenz wurde gefordert, geschlechtsspezifische Daten von wissenschaftlichen und bildungspolitischen Institutionen erheben zu lassen. Des Weiteren forderten sie eine Förderung der Präsenz von Frauen in den Ausschüssen, in denen Entscheidungen über die Besetzung von Lehrstühlen der Fakultäten und des Forschungspersonals getroffen werden sowie in den Wahlgremien für hoch angesehene Positionen in Forschung und Lehre. 
Im Dezember 2001 wurde die Asociación Española de Mujeres Científicas y Tecnólogas, AMIT (Spanische Vereinigung der Frauen in Naturwissenschaften und Technologie) gegründet. Zu den Gründungsmitgliedern gehören Forscherinnen und Professorinnen, die in verschiedenen wissenschaftlichen Disziplinen arbeiten. Sie beanspruchen eine gerechtere Anerkennung der Leistungen von Frauen. Unter ihnen finden sich die Biomedizinerin Flora de Pablo, die Philosophin Eulalia Pérez Sedeño, die Chemikerin Caridad Ruiz, die Physikerin Pilar López-Sancho und die Soziologin Maria Ángeles Durán. Diese bekannten Wissenschaftlerinnen und die steigende Anzahl von Mitgliedern der AMIT begründen einen sozialen Raum für Frauen und sie stellen gleichzeitig eine Bühne zur Verfügung, auf der Rechte gefordert werden können. Die Vereinigung entwickelte sich zum „follow-up" der Rechtsnormen, die die Behörden nach und nach genehmigten, um den geschlechtsspezifischen Bias in den Wissenschaften mit Blick auf den Anteil von Frauen an den Lehrstühlen und unter dem Forschungspersonal in Spanien $\mathrm{zu}$ überwinden. Im gleichen Jahr gründete der Consejo Superior de Investigaciones Científicas, CSIC (Oberster Rat der wissenschaftlichen Forschung) die größte öffentliche Organisation Comisión Mujer y Ciencia (Kommission für Frauen und Wissenschaft), die sich der Rolle der Frau in der Forschung Spaniens widmet. Das Ziel dieser Kommission ist es, die Präsenz von Frauen auf allen Personalebenen und in den Ausschüssen zu untersuchen, zu fördern und zu sichern.

2002 wurde auch eine Untersuchung über Frauen in der CSIC durchgeführt, die verschiedene Disziplinen untersuchte, um die Präsenz von Wissenschaftlerinnen in jedem dieser Bereiche und ihre persönlichen Erfahrungsberichte zu berücksichtigen. In dieser Forschungsarbeit wurde nicht ausdrücklich die Diskriminierung während des beruflichen Werdegangs von Frauen nachgewiesen, aber es zeigte sich, dass sie eine Minderheit in allen Fächern bildeten (Fernández Vargas und Santesmases 2002). 34,14 Prozent der wissenschaftlichen Mitarbeiter des CSIC waren Frauen und 21,99 Prozent der Forschungsprofessuren wurden von Frauen eingenommen - also der Ebene, die eine gehobene Anerkennung mit sich bringt. In einer längeren Perspektive zeigt sich, dass sich während der letzten Jahrzehnte die Präsenz von Frauen unter den wissenschaftlichen Mitarbeitern dieser Körperschaft kaum geändert hat und in einigen Arbeitsbereichen sogar gesunken ist (Alcalá 1995).

Eine Initiative des Abgeordnetenhauses hat im November 2002 empfohlen, Daten über die Beiträge der spanischen Wissenschaftlerinnen und über ihre berufliche und akademische Situation zu sammeln. Das spanische Parlament erkannte die Notwendigkeit an, die Situation von Frauen in Wissenschaft und Technik zu verbessern. Die spanische Stiftung für Wissenschaft und Technologie (FECYT) wurde beauftragt, diese Initiative auszuarbeiten. Sie gab einen Bericht in Auftrag, der 2005 veröffentlicht wurde. Er enthielt Empfehlungen zur Auswertung und Nachkontrolle der geschlechtsspezifischen Daten und schlug vor, ein Referat für Frauen und Wissenschaft im Ministerium für Bildung und Wis- 
senschaft zu gründen. Dieses Referat soll die Datenerhebungen und ihre Überprüfung pflegen sowie Maßnahmen zur Aufhebung vorhandener Ungleichheiten zwischen den Geschlechtern in der wissenschaftlichen und akademischen Welt und positive Strategien zur Förderung der Sichtbarkeit von Frauen in Wissenschaft und Forschung kontrollieren (Expert Group 2005).

Im selben Jahr wurde auch eine Verordnung der Regierung erlassen, mit der die Gleichstellung von Frauen und Männern gefördert werden sollte. Darin war u.a. juristisch festgelegt, dass die Personalleitungsstelle der zentralen Verwaltung (dem allgemeinen Organ der staatlichen Beamten) eine paritätische Zusammensetzung haben soll. Auch wurde die Gründung einer Abteilung für „Frauen und Wissenschaft" vereinbart, ,um die Situation von Frauen in Forschungseinrichtungen in Angriff zu nehmen und um ihre Präsenz in ihnen zu verbessern".1

Somit scheint das Bewusstsein für den Gender Bias, den die Zugangskriterien für Frauen auf Forschungspositionen und Hochschullehrerstellen an den spanischen Universitäten vorweisen und vorgewiesen haben, zugenommen zu haben. Dieses erhöhte Bewusstsein zeigt sich auch in einer Regierungspolitik, die Gleichstellung durch verschiedene politische und rechtliche Mechanismen fördert. Als eine Folge sind die Daten über den Prozentsatz von Frauen und Männern, die das spanische System der Wissenschaft und Technik erhebt, zunehmend nach Geschlechtern aufgeschlüsselt. Nichtsdestotrotz bleiben viele relevante Zahlen immer noch im Verborgenen oder sind nur schwer zu erheben. Aber das nationale und internationale Bewusstsein über Voreingenommenheit und Diskriminierung aufgrund des Geschlechts scheinen doch nicht auszureichen, um ungerechte Situationen zu überwinden. Eine mögliche Verbesserung könnte in der Forschung über Beiträge von Frauen zu zeitgenössischen Wissenschaftsfragen liegen. Gleichzeitig sollten längerfristig die Wege der Frauen in der Wissenschaft untersucht werden, so dass nach und nach die kulturellen Räume des Handelns und der Autorität, die gewonnen oder wieder verloren wurden, in ihrer historischen Entwicklung sichtbar werden.

Eine kontinuierlich zunehmende Beteiligung von Frauen im öffentlichen, wissenschaftlichen und beruflichen Leben in Spanien wurde bisher nicht über lange Zeiträume beobachtet. Dies lässt vermuten, dass androzentrische kulturelle Werte einen starken Einfluss auf die Gesellschaft haben. Der spanische akademische Feminismus widmet sich daher zwei miteinander verbundenen Themenkomplexen: einerseits der kritischen Kontrolle von Statistiken, die zwar sehr leichte Verbesserungen aufzeigen, aber auch rückgängige Zahlen der berufstätigen Frauen und der akademischen, weiblichen Gelehrten, andererseits der kontinuierlichen Forschung über Beiträge von Frauen in der Geschichte und der Wissenschaft.

1 Orden PRE / 525 / 2005, Boletín Oficial del Estado de 8 de marzo de 2005. 


\section{Feminismus und Wissenschaftskulturen}

Für die historische Forschung sowie die qualitative soziologische Forschung über Wissenschaftlerinnen und Akademikerinnen in Spanien gibt es einige besondere Arbeits- und Diskussionsbereiche. Eine sehr wichtige Anerkennung der feministischen Bewegung in der Wissenschaft (auch in finanzieller Hinsicht) war 1996 die Aufnahme der Frauen- und Geschlechterforschung in den Nationalen Forschungsplan, einer Einrichtung des Ministeriums für Bildung, Wissenschaft und Forschung, das den Staatshaushalt für die Forschung in Spanien verwaltet. Die Integration der feministischen Forschung in die nationalen Forschungsprogramme erweiterte ihre Anerkennung und Sichtbarkeit in der akademischen Welt. Das 1983 gegründete Instituto de la Mujer (Fraueninstitut) hat seitdem ein eigenes Budget für diese Forschung und einen eigenen Verlag. Außerdem wurden Seminare und Institute für Frauenforschung an den Universitäten von Barcelona, der Autonomen Universität von Madrid und der Universität des Baskenlandes gegründet, viele andere Universitäten folgten (Ballarín, Gallego / Martínez 1995).

In den akademischen Disziplinen in Spanien sind Medizin und Gesundheitstechnik weiter verbreitet und Gegenstand interessanterer Forschungsarbeiten als die der Naturwissenschaften und der Geschlechter. Einige weibliche Absolventen der Medizin erreichten akademischen, sozialen und politischen Einfluss und bildeten einen Teil der feministischen Bewegung in Spanien seit 1970 (Scanlon 1986). Die feministische Kritik zeigte zum Beispiel für die Medizinforschung die Voreingenommenheit gegen Frauen auf, die besonders in der unterschiedlichen Behandlung, die Frauen in den medizinischen Lehrbüchern erhielten, deutlich wurde (wie z.B. die Thematisierung der Geschlechtsorgane von Männern und Frauen). Aber nicht nur in der Medizin, sondern auch in der Rechtswissenschaft, bei der es um Sexualdelikte oder um Recht und Wirtschaft von Frauen und Familien geht, wurden Vorurteile deutlich (Gallego Méndez 1983). In den Universitäten, den Gesundheitszentren und Krankenhäusern arbeiteten und arbeiten immer noch viele Frauen daran, ihre Berufsrolle gleichberechtigt neben ihren männlichen Kollegen auszuführen.

Carmen Alvarez-Ricart hat vor mehr als zwei Jahrzehnten eine bahnbrechende Studie über spanische Ärztinnen veröffentlicht. Sie beschrieb die Arbeit der Pionierinnen auf diesem Gebiet und deren historische Vorgeschichte. Die katholische Kirche erlaubte Nonnen die Ausgabe medizinischer Präparate, eine Tatsache, die den hohen Anteil von Frauen in der Pharmazie in Spanien erklären mag. Dies legen zumindest jene Studien nahe, die sich mit weiblichen Ordensgemeinschaften im Mittelalter und in der Neuzeit beschäftigen. Diese Gemeinschaften waren soziale Räume, in denen Frauen Wissen produzierten und ihre Interessen entwickelten (Álvarez-Ricart 1988). Die Präsenz von Frauen im Apothekerberuf wurde von anderen Autoren mit der traditionellen weiblichen Pflege von Patienten und ihren Familien verbunden (de Miguel/Salcedo, 1987). Des Weiteren ha- 
ben medizinische Kongresse die Gesundheits- und Geschlechterforschung integriert, woran spanische Expertinnen kontinuierlich beteiligt sind (Cabré / Ortiz Gómez 2007). Die Forschung über Frauen und Gesundheit zeigen den Gender Bias in der medizinischen Praxis (Valls-Llobet 2006).

Zurzeit beschäftigen sich Forscherinnengruppen aus verschiedenen Disziplinen mit der Geschlechter- und Wissenschaftsforschung in Spanien. Hier wird besonders auf die weiblichen Ausdrucksformen geachtet, weil es hauptsächlich Frauen sind, die zum heutigen Erkenntnisstand zum Gender Bias beitragen. Weibliche Philosophen, Ärzte und eine wachsende Gruppe von experimentellen Wissenschaftlerinnen verfügen über soziale Anerkennung und akademischen Respekt im Bereich der Geschlechterforschung, experimentellen Wissenschaften und Medizin. Diese zunehmende Anzahl von Expertinnen beschäftigt sich mit Forschungen, die von der epistemischen und kulturellen Grundlage der feministischen Kritik über die Analyse scheinbar ahistorischer Diskurse und wissenschaftlicher Bildung aus der Geschlechterperspektive bis hin zu Erfahrungsberichten der Wissenschaftlerinnen selbst reichen (Barral / Magallón / Miqueo / Sánchez 1999).

An vielen Universitäten und Forschungsinstituten werden Studien über Geschlecht und Gesundheit in der Geschichte der Medizin und der medizinischen Wissenschaft durchgeführt. Arbeiten sowohl über Heilerinnen und Hebammen als auch über Frauen in der Medizin in der heutigen Zeit zeigen die Forschungsschwerpunkte in Spanien, die seit den 1980er Jahren entwickelt wurden. Die kulturellen Wirkungen dieser Forschungen werden nicht nur durch die Anzahl der Studien deutlich, sondern auch durch deren Einbeziehung in die allgemeine Geschichte der Frauen in Spanien (Ballarín / Martínez 1995; Borderías 2008).

Ein detaillierter Überblick über das Geschlecht und die Medizin in der Geschichte wurde von Teresa Ortiz veröffentlicht, eine ausgezeichnete Einführung in diese Wissensgebiete über Medizin, Wissenschaft und Geschlecht (Ortiz Gómez 2006, 1987). Die systematische und wissenschaftliche Forschung von Isabel Delgado zur Geschichte der Geschlechtschromosomen von Pflanzen und Fruchtfliegenkäfern und die herausragende Arbeit von Marta González über die wissenschaftlichen Definitionen der weiblichen Sexualität sind verbunden mit anderen Studien zum reproduktiven Körper und zur künstlichen Befruchtung. In diesem Bereich ist die Philosophin Ana Sanchez aus Valencia eine Pionierin der Forschung gewesen. Aber auch Arbeiten über „Liebe“ als Verursacherin von Ungleichheit im Kontext der Forschung über Emotionen und Geschlecht müssen hier erwähnt werden (Delgado Echeverría 2007; González 2009; Sánchez Torres 2005; Esteban / Medina /Távora 2005).

Die genannten Werke sind nur einzelne Beispiele. Um detailliertere Informationen zu finden, reicht es inzwischen, den Namen der Autorinnen in ein Suchportal im WorldWideWeb einzugeben, wodurch lange Listen entstehen, in denen die Welt der Frauen sich in jedem Text neu bildet. Dieses Netz basiert auf kollektiver Arbeit, die auf Gruppenstrategien und Verbindungen zwischen ein- 
zelnen Gruppen beruht. Es war genau diese kollektive Anstrengung, die es heute ermöglicht, die intellektuellen und sozio-akademischen Wege der feministischen Forschung in der Wissenschaft aufzuzeigen. Einige sind das Produkt einer fortlaufenden Reflexion über das weibliche Anderssein und ihrer Genealogie.

Kollektives Handeln von Frauen war und ist, so lässt sich vermuten, sinnvoll und notwendig. Dies legen zumindest neue politikwissenschaftliche Arbeiten nahe, die die Rationalität kollektiven Handelns gerade für marginalisierte Gruppen betonen, die so, durch gegenseitige Unterstützung und Schutz, ihre jeweiligen Ziele leichter erreichen können. Auf diese Weise könnte auch erklärt werden, warum die meisten spanischen feministischen Publikationen über die Wissenschaften kollektiv erarbeitet wurden, und zwar weil sich über diese Arbeitsform auch Inhalte ausdrücken. Das Kollektive ist eine Form, Arbeiten zu präsentieren, die sich überlagern und dann in einem kooperativen Projekt zusammenlaufen, um gemeinsame Interessen in einer akademischen Umgebung zu entwickeln (Magallón / Barral / Delgado / Fernández / Miqueo 2003; Miqueo /Tomás /Tejero / Barral / Fernández /Yago 2001; Cabré / Ortiz Gómez 2008; Roldán / González 2008).

Einige Forschungsgruppen über Geschlecht, Wissenschaft, Medizin und Philosophie in Spanien koordinieren und kontaktieren sich über gemeinsame Arbeitsprozesse, organisieren Treffen und erarbeiten Fallstudien, an denen diejenigen Autorinnen und Autoren beteiligt sind, deren Themen konvergieren und die sich gegenseitig sichtbar machen. Durch diese Form der Arbeit wird Autorität verteilt und gemeinsam genutzt. Die Wirksamkeit dieser Strategie liegt auf der Hand, besonders wenn man die entsprechenden Beiträge in den regelmäßig erscheinenden Zeitschriften zusammenzählt. Außerdem werden diese Veröffentlichungen, und das ist vielleicht ihr größter Wert, in gemeinsame Arbeitsquellen, Unterrichtsmaterialien und (spanische) Bibliographien verwandelt. Die Frage der Sprache ist relevant, denn man scheint gelegentlich zu einseitig auf ausländische, besonders englische Literatur zurückgegriffen zu haben, vermutlich, um entsprechende Sprachkenntnisse und den Zugang zur aktuellen ausländischen Forschung zu fördern. Allerdings scheint es sinnvoll, in die Bibliographien der Grund- und Aufbaustudiengänge bis zu einem gewissen Grad spanische oder übersetzte Arbeiten mit aufzunehmen, um die jeweiligen Narrative leichter zugänglich zu machen und gleichzeitig die Studierenden dazu zu befähigen, die jeweilige Fachterminologie zu de- und rekonstruieren.

Die Arbeiten, die Wissenschaftlerinnen über Geschlecht und Wissenschaften verfasst haben, konzentrieren sich auf die „weibliche akademische Genealogie“ jener Frauen, die zu naturwissenschaftlichem und medizinischem Wissen und zur jeweiligen Praxis sowie zur Erforschung des geschlechtsspezifischen Bias in den jeweiligen Fächern beigetragen haben. Diese Arbeiten werden ergänzt durch Studien teilweise noch sehr junger Kolleginnen aus der Soziologie und Anthropologie, die sich aus geschlechtergeschichtlicher Perspektive mit Fragen der 
Körpergeschichte und der feministischen Philosophie bzw. der Erkenntnistheorie beschäftigen. In der Pädagogik wiederum wurden, meist von Lehrerinnen der Sekundarschulen, die ersten Schriften zum Thema „Bildung und ihre geschlechtsspezifische Voreingenommenheit" erstellt.

Die Geschichtswissenschaftlerinnen der Medizin und die Mediävistinnen, Soziologinnen und Philosophinnen vereinigen und koordinieren sich immer öfter, um dauerhafte Kontakte zu knüpfen, damit die Artikulation der Wissenschaftsforschung und Geschlechterstudien in Spanien in Einklang gebracht werden kann, sich ihr Anerkennungsgrad verbreitet und ihre Wirkungsmacht weiter generiert und verbreitet wird.

\section{Schlussfolgerung}

Die vielen Publikationen der letzten Jahrzehnte zeigen Vielfalt und Reichtum der Frauen- und Geschlechterforschung in Spanien sowie eine Bereitschaft, Vernetzungen zwischen den Disziplinen zu implementieren und thematische Schnittstellen aufzuzeigen, belegen also intellektuelle Berührungspunkte und akademischen Aktivismus sowohl in der Analyse als auch in der Forschung. Diese Entwicklung wurde als Zusammenbruch oder auch als Verschiebung sowohl der disziplinären Schranken als auch des eigenen Geschlechts wahrgenommen. Und so wäre dies vielleicht die wichtigste Schlussfolgerung für die Geschlechterstudien in Spanien insgesamt: der explizit formulierte Wunsch nach wachsender fächerübergreifender Koordination und die Tendenz, diese Koordination zu institutionalisieren.

Diese Analyse lässt sich leicht mit Zahlen untermauern. Die staatliche Setzung neuer Rechtsnormen und deren juristische Überwachung reichen bei weitem nicht aus, denn androzentrische und geschlechtsdiskriminierende Kulturen sind sehr viel schwieriger zu verändern als Gesetzestexte. Es sind letztlich die menschlichen Körper, die Identitäten stabilisieren oder verändern. Und zwischen diesen Körpern einerseits und den kulturellen Normen andererseits sind jene geschlechtsspezifischen Statistiken über den wissenschaftlichen Beitrag von Frauen und Männern in Spanien ebenso angesiedelt wie die kulturwissenschaftlichen und feministischen Studien über Wissenschaft, Biologie und den Körper. Beides, Körper und Zahlen zu denaturalisieren ist die Aufgabe der feministisch inspirierten Wissenschaft in Spanien.

Aus dem Spanischen von Miriam Seemann Redaktionelle Bearbeitung Kirsten Heinsohn und Stefanie Schüler-Springorum 


\section{Literatur}

Alcalá, Paloma (1995): Españolas en el CSIC: presencia y status de las mujeres en la investigación científica española: el CSIC 1940-1993. In: Ortiz Gómez, Teresa / Becerra Conde, Gloria (1996) (Hrsg.): Mujeres de ciencias. Mujer, feminismo y ciencias naturales, experimentales y tecnológicas. Granada, $61-73$.

Alcalá Cortijo, Paloma (2002): Enseñando a perder. In: Emakunde, Nr 49, 30-33.

Alcalá Cortijo, Paloma (2006): A ras de suelo. Situación de las mujeres en las instituciones científicas. In: Ciencia, Tecnología y Género en Iberoamérica. Madrid, 89-98.

Alcalá, Paloma (2008): Cuéntame cómo te ha ido: de mujeres, ciencia y democracia, 1970-2006. In: Isegoría, Nr. 38, 187-195.

Álvarez Ricart, Carmen (1988): La mujer como profesional de la medicina en la España del siglo XIX. Barcelona.

Ballarín, Pilar / Gallego, M. Teresa / Martínez, Isabel (1995): Los estudios de las mujeres en las universidades española 1975-1995. Libro Blanco. Madrid.

Barral, María José / Magallón, Carmen / Miqueo, Consuelo / Sánchez, María Dolores (1999) (Hrsg.): Interacciones ciencia y género: discursos y prácticas científicas de mujeres. Barcelona.

Borderías, Cristina (2008) (Hrsg.): La historia de las mujeres: perspectivas actuales. Barcelona.

Cabré Montserrat/ Ortiz Gómez, Teresa (2001) (Hrsg.): Sanadoras, matronas y médicas en Europa, siglo XII-XX. Barcelona.

Cabré, Montserrat/Ortiz Gómez, Teresa (2007): Relaciones entre la historia de la medicina y la investigación en salud y género. In: Campos, Ricardo / Montiel, Luis/Huertas, Rafael (Hrsg): Medicina, ideología e historia en España (siglos XVI-XXI). Madrid, 627-634.

Cabré, Montserrat / Ortiz Gómez, Teresa (2008) (Hrsg.): Significados científicos del cuerpo de mujer, número monográfico de Asclepio.Vol. LX, No. 1.

Capel, Rosa M. (1982): El trabajo y la educación de la mujer en España 1900-1930. Madrid.

Carabaña, Julio (1984): Modesto intento de interpretación de las tasas femeninas de escolaridad. In: Revista de Educación, Nr. 275, 19-41.

Carabaña, Julio (1997): La evolución de las desigualdades educativas por clases sociales en España según la Encuesta Sociodemográfica, 1907-1976, comunicación presentada a la VI Conferencia de Sociología de la Educación, Jaca, septiembre de 1997.

De Miguel, Jesús / Salcedo, Juan. (1987): La profesión farmacéutica. Madrid.

Delgado Echeverría, Isabel (2007): El descubrimiento de los cromosomas sexuales. Madrid.

Durán, María Ángeles (1972): El trabajo de la mujer en España. Un estudio sociológico. Madrid.

Esteban Galarza, Mari Luz / Medina Doménech, Rosa /Távora Rivero, Ana (2005): ¿Por qué analizar el amor? Nuevas posibilidades para el estudio de las desigualdades de género. In: Díez Mintegui, Carmen / Gregorio Gil, Carmen (Hrsg.): Cambios culturales y desigualdades de género en el marco local-global actual. X Congreso de Antropología. Sevilla, 207-223.

Férnandez Vargas, Valentina / Santesmases, María Jesús (2002) (Hrsg.): Ciencia y tecnología en el CSIC. Una visión de género. Número monográfico de la revista Arbor (número 679-680, Tomo CLXXII, Juli-August 2002).

Flecha, Consuelo (1996): Las primeras universitarias en España. Madrid.

Gallego Méndez, Teresa (1983): Mujer, Falange y franquismo. Madrid.

García Dauder, Silvia (2005): Psicología y feminismo: historia olvidada de mujeres pioneras en psicología. Madrid.

González Marta (2009): Biomedicalización: redefiniciones tecnocientíficas de la sexualidad femenina. In: Ensayos sobre bioética. Salamanca,153-166. 
Grupo de expertas (Palomá Alcalá, María Bordons, María Luisa García de Cortázar, Marina Griñón, Ana Guil, Ana Muñoz, Eulalia Pérez Sedeño, María Jesús Santesmases) (2005): Mujer y ciencia. La situación de las mujeres investigadoras en el sistema español de ciencia y tecnología. Madrid.

Magallón Portolés, Carmen (1998): Pioneras españolas en las ciencias. Las mujeres del Instituto Nacional de Física y Química. Madrid.

Magallón Portolés, Carmen / Barral Morán, María José / Delgado Echeverría, Isabel / Fernández Turrado, Teresa / Miqueo Miqueo, Consuelo (2003): Del análisis crítico a la autoridad femenina en la ciencia. In: Feminismo/s: revista del Centro de Estudios sobre la Mujer de la Universidad de Alicante, Nr. 1, 195-216.

Miqueo, Consuelo /Tomás, Concepción / Tejero, Cruz / Barral, María José / Fernández, Teresa /Yago, Teresa (2001) (Hrsg.): Perspectivas de género en salud. Fundamentos científicos y socioprofesionales de diferencias sexuales no previstas. Madrid.

Muñoz Repiso, Mercedes (1988): La presencia de las mujeres en el sistema educativo. Madrid.

Núñez, Clara Eugenia (1992): La fuente de la riqueza: Educación y desarrollo económico en la España contemporánea. Madrid.

Ortiz Gómez, Teresa (1987): Médicos en la Andalucía del siglo XX. Número, distribución, especialismo y participación profesional de la mujer. Granada.

Ortiz Gómez, Teresa (2006): Medicina, historia y género. 130 años de investigación feminista. Oviedo.

Pérez Sedeño, Eulalia (2005): La situación de las mujeres en el sistema educativo de ciencia y tecnología en España y su contexto internacional.

Pérez Sedeño, Eulalia / Kiczkowski, Adriana (2010): Género y astronomía en España: Un universo por descubrir. Madrid.

Roldán, Concha / González, Marta (2008) (Hrsg.): Feminismos: nuevas tendencias. Número monográfico. In: Isegoría Nr. 38.

Salido, Olga (1996): La movilidad ocupacional femenina en España: una comparación por sexo. Tesis doctoral, Madrid, Universidad Complutense.

Sánchez Torres Ana (2005): Las tecnologías de reproducción asistida y sus metáforas. In: Arbor, Nr. $716,523-530$.

Santesmases, María Jesús (2001): Mujeres científicas en España, profesionalización y modernización social. Madrid.

Scanlon, Geraldine (1986): La polémica feminista en la España contemporánea. Madrid.

Valls-Llobet, Carmen (2006): Mujeres invisibles. Barcelona.

Zulueta, Carmen de (1984): Misioneras, feministas, educadoras. Historia del Instituto Internacional. Madrid.

Zulueta, Carmen de / Moreno, Alicia (1993): Ni convento ni college: La Residencia de Señoritas.. Madrid. 